\title{
A model of the anchoring effect in dichotomous choice valuation with follow-up
}

\author{
Sandra Lechner* \\ University of Konstanz,
}

\author{
Anne Rozan ${ }^{\dagger}$ \\ University of Strasbourg, \\ BETA
}

\author{
François Laisney \\ University of Strasbourg, \\ ZEW, Mannheim
}

\begin{abstract}
This paper focuses on modelling and estimating the starting point bias in closed-ended follow-up questions, where several bids are presented successively, depending on previous answers. Although the contingent valuation literature took off in the last decade, there is only one study modelling the starting point bias. We propose a new modelling of this anchoring effect based on the assumption the first proposed bid has a direct influence on the individual's willingness-to-pay, i.e respondents modify their willingness-to-pay when presented with the first bid just before they answer the first question. Monte Carlo results support the specification of our model. An application is provided based on data from a contingent valuation study conducted concerning air quality in Strasbourg.
\end{abstract}

JEL classification: C15, C25, D1, Q0

Keywords: Contingent valuation, willingness-to-pay, binary-choice, anchoring effect

*Department of Economics, Box D 124, University of Konstanz, 78457 Konstanz, Germany. Phone (+49)7531 88 3214. Fax (+49) 753188 4450, E-mail : Sandra.Lechner@uni-konstanz.de.

†BETA UMR 7522, Université Louis Pasteur, 61 Avenue de la Forêt Noire, 67085 Strasbourg Cedex, France. Phone $(+33) 3.90 .24 .21 .06$, Fax $(+33) 3.90 .24 .20 .71$, E-mail: rozan@cournot.ustrasbg.fr

¥BETA THEME, Université Louis Pasteur, Strasbourg, and ZEW, Mannheim, Postfach 103443, D-68034 Mannheim, Germany. Phone (+49) 6211235 154, Fax (+49) 6211235 225, E-mail: laisney@zew.de. The first author wishes to thanks the ZEW for its hospitality and Michael Lechner for helpful comments. 


\section{Introduction}

The contingent valuation method (CVM) has become a standard approach for valuing nonmarket "goods", with environmental quality as a typical example. As the method is controversial (see Hausman, 1993), participants in the N.O.A.A. Panel have proposed some recommendations (see Arrow et al., 1993). Concerning the elicitation technique, they advocated the single referendum format, because respondents do not have to reveal their willingness-to-pay (WTP) directly, as in the open-ended question. However, as this method can be very inefficient, a suggestion for increasing efficiency has been to resort to a survey with "sequential bids", where at each step of the questioning, the proposed bid is either greater or lower than the previous one, depending on the answer to the last question (see, e.g, Cameron and James, 1987, Kanninen, 1995 and Langford et al., 1996).

This approach is appealing because it yields more precise information than the single referendum. Its obvious drawback lies in the corresponding starting point bias or anchoring effect, caused by the respondents' reactions to the bids presented. Therefore, when accepting or refusing the bid, respondents take into account their private information, as well as the information contained in the bid itself, interpreting the proposed bid as an amount which should be paid rather than as an amount selected to maximise the efficiency of the survey design. This particular behavior is described by Mitchell and Carson (1989, p.240): "confronted with a dollar figure in a situation where he is uncertain about an amenity's value, a respondent may regard the proposed amount as conveying an approximate value of the amenity's true value and anchor his WTP amount on the proposed amount". In other words, instead of comparing their WTP with the proposed bid, respondents incorporate the announced bid in thinking of their WTP and update their WTP. This phenomenon leads to biased estimation of the mean and the standard deviation of the WTP.

While several studies (e.g. O'Connor et al.,1999, Greene et al., 1998) found strong anchoring effects induced by dichotomous choice with follow-up, only Herriges and Shogren (1996) proposed a model of the starting point bias. Yet their model only accounts for the effect of the first bid. Here, we propose a model based on the hypothesis that the anchoring effect is induced by all successive bids of the questionnaire, and not only by the first bid. 
The paper is organized as follows. Section 2 describes the model, Section 3 presents simulation results of the estimation of the anchoring effect. Section 4 provides an application of the starting point bias based on data from a contingent valuation study conducted in Strasbourg. Section 5 concludes.

\section{The model}

The starting point or anchoring phenomenon appears in situations where respondents are uncertain and consider the proposed bid as information on the amount which should be paid. Cameron and Quiggin (1994) suppose that, before people decide to participate in the survey, they have no preestablished WTP, and that they build it through the follow-up questioning, taking into account their preferences and the proposed bids. Instead of comparing their WTP with the proposed bid, they combine both and finally compare the revised WTP with the proposed bid, i.e. respondents anchor their answers on the proposed bid. Ignoring this phenomenon leads to biased estimation of the mean and the standard deviation of the WTP. An illustration of these biases is given in the CV study about air quality conducted by Rozan (1999). A closed-ended questionnaire with follow-up was conducted, where people were randomly allocated to one of two versions of the questionnaire. ${ }^{1}$ The results showed a significant difference in WTP w.r.t. the version of the questionnaire: respondents to the high version revealed a higher WTP than respondents to the low version.

Our specification is based on the model of Herriges and Shogren (1996). Firstly, we shortly describe the latter. Herriges and Shogren assume that respondents anchor their WTP amount on the first proposed bid when responding to the second rather than to the first question. In other words, they suppose that the response to the second question depends not only on the individual's WTP (W), but also on the

\footnotetext{
${ }^{1} \mathrm{~A}$ low version began with an amount of $50 \mathrm{FF}$ (around $7.62 €$ ) and a high version with an amount of $2000 \mathrm{FF}$ (around $304.90 €)$. $(1 €=6,55957 \mathrm{FF}$ )
} 
first proposed bid in the following way:

$$
\begin{aligned}
& R_{1}\left(b_{i}^{1}\right)=\left\{\begin{array}{l}
1, \text { if } f(X, \theta, \varepsilon)=W \geq b_{i}^{1}, \\
0, \text { otherwise. }
\end{array}\right. \\
& R_{2}\left(b_{i}^{2}\right)= \begin{cases}1, \text { if }(1-\lambda) W+\lambda b_{i}^{1}=\tilde{W} \geq b_{i}^{2}, \\
0, \text { otherwise. }\end{cases}
\end{aligned}
$$

where $R_{i}\left(b_{i}^{j}\right)$ is the $j$ th response on the $j$ th proposed bid, and $f($.$) is a function$ which depends on a vector $X$ of individual characteristics (income, age, education, transportation mode, ...), on a vector of unknown parameters $\theta$ and on a zero mean random variate $\varepsilon$. $\lambda$ measures the anchoring effect and is located in the interval $[0,1]$. Therefore in one extreme case, $\lambda=0$, there is no anchoring effect, because $\tilde{W}=W$ and in the other extreme case, $\lambda=1$, the individual ignores his WTP and replaces it with the proposed bid, $\tilde{W}=b_{i}^{1}$.

Our model departs from this basic model in two ways. Firstly we suppose that the first proposed bid has an immediate influence on the individual's WTP, so that the respondents modify their WTP before they answer the first question of the study. It seems odd to assume that the first bid has an influence on the second answer only, and not on the first one, as Herriges and Shogren (1996) do. Secondly, we assume that the anchoring effect is induced by all bids of the questionnaire. It seems unrealistic to consider that the anchoring effect is only induced by the first bid. Respondents do not only compare their revised WTP with the first proposed bid, but the same mechanism is at work at each step of the questionnaire. However, we impose the restriction that people consider all bids equivalently, in other words, they attach the same anchoring effect to each proposed bid. ${ }^{2}$

We consider a double bounded dichotomous choice model and assume people have an initial constant WTP, i.e. we assume people have a preestablished WTP before they are willing to participate in the survey. Let $Y_{0 i}^{*}$ be the initial WTP. We assume that its conditional expectation given the individual's characteristics $X_{i}$ is linear in those and thus posit the regression model:

$$
Y_{0 i}^{*}=X_{i} \beta+\varepsilon_{i}, \quad \text { where } \quad \mathrm{E}\left[\varepsilon_{i} \mid X_{i}\right]=0 .
$$

\footnotetext{
${ }^{2}$ This hypothesis will clearly need to be tested against the alternative where the weights of the follow-up bids differ.
} 
When confronted with the first proposed bid $b_{i}^{1}$, the individual revises his WTP according to:

$$
\begin{aligned}
Y_{1 i}^{*} & =(1-\lambda) Y_{0 i}^{*}+\lambda b_{i}^{1}+\eta_{i}, \\
& =(1-\lambda) X_{i} \beta+\lambda b_{i}^{1}+(1-\lambda) \varepsilon_{i}+\eta_{i}, \\
& =(1-\lambda) X_{i} \beta+\lambda b_{i}^{1}+u_{1 i} .
\end{aligned}
$$

Since the joint distribution of $\varepsilon_{i}$ and $\eta_{i}$ is not identified, in the absence of an observation directly related to $Y_{0 i}^{*}$, we define $u_{1 i}=(1-\lambda) \varepsilon_{i}+\eta_{i}$ as the error term of the revised WTP, with variance equal to $\sigma_{1}^{2}=(1-\lambda)^{2} \sigma_{\varepsilon}^{2}+\sigma_{\eta}^{2}+2(1-\lambda) \sigma_{\varepsilon \eta}$. It seems reasonable to assume that the anchoring parameter $\lambda$ is located in the interval $[0,1]$. Instead of comparing their WTP with the proposed bid, respondents combine both and compare the revised WTP, $Y_{1 i}^{*}$, with the proposed bid $b_{i}^{1}$, in the following way:

$$
R_{1}\left(b_{i}^{1}\right)= \begin{cases}1, & \text { if }(1-\lambda) X_{i} \beta+\lambda b_{i}^{1}+u_{1 i} \geq b_{i}^{1}, \\ 0, & \text { otherwise }\end{cases}
$$

where $R_{1}\left(b_{i}^{1}\right)$ is the first response on the first proposed bid. We also suppose that the anchoring phenomenon is remains active for subsequent bids, with the same parameter $\lambda$, and that the revised WTP for a two-bid model is

$$
\begin{aligned}
Y_{2 i}^{*} & =(1-\lambda) Y_{1 i}^{*}+\lambda b_{i}^{2}+u_{2 i}, \\
& =(1-\lambda)^{2} X_{i} \beta+\lambda(1-\lambda) b_{i}^{1}+\lambda b_{i}^{2}+(1-\lambda) u_{1 i}+u_{2 i},
\end{aligned}
$$

where

$$
\left[\begin{array}{l}
u_{i 1} \\
u_{i 2}
\end{array}\right] \sim N\left(\left[\begin{array}{l}
0 \\
0
\end{array}\right],\left[\begin{array}{cc}
\sigma_{1}^{2} & \sigma_{12} \\
\sigma_{12} & \sigma_{2}^{2}
\end{array}\right]\right)
$$

Then, as in the one-bid model, people combine their revised WTP, $Y_{1 i}^{*}$, with the second proposed bid, $b_{i}^{2}$, and compare the new revised WTP, $Y_{2 i}^{*}$, with the second proposed bid, $b_{i}^{2}$, in the following way:

$$
R_{2}\left(b_{i}^{2}\right)= \begin{cases}1, & \text { if }(1-\lambda)^{2} X_{i} \beta+\lambda(1-\lambda) b_{i}^{1}+\lambda b_{i}^{2}+(1-\lambda) u_{1 i}+u_{2 i} \geq b_{i}^{2} \\ 0, & \text { otherwise }\end{cases}
$$

where $R_{2}\left(b_{i}^{2}\right)$ is the second response, on the second proposed bid. Let $v$ be the error 
term of the two-bid model, defined as $v=(1-\lambda) u_{1 i}+u_{2 i}$, with variance equal to $\sigma_{v}^{2}=(1-\lambda)^{2} \sigma_{1}^{2}+\sigma_{2}^{2}+2(1-\lambda) \sigma_{12}$.

In this model we have five structural parameters, $\beta, \lambda, \sigma_{1}, \sigma_{2}$ and $\sigma_{12}$ the covariance between the errors terms. Only four of these are separately identified and we choose to estimate $\beta_{i}, \lambda, \sigma$ and $\sigma_{v}$, where $\sigma$ corresponds to the restriction $\sigma_{1}=\sigma_{2} \cdot{ }^{3}$ Using this identifying restriction, the model is estimated with maximum likelihood. The expression of the likelihood is given in the appendix.

$$
\begin{aligned}
& R_{2}\left(b_{i}^{2}\right)= \begin{cases}1, & \text { if } Y_{2 i}^{*} \geq b_{i}^{2}, \\
0, & \text { otherwise, }\end{cases} \\
& R_{1}\left(b_{i}^{1}\right)= \begin{cases}1, & \text { if } Y_{1 i} \geq b_{i}^{1}, \\
0, & \text { otherwise }\end{cases}
\end{aligned}
$$

\section{Monte Carlo Experiment}

The construction of the Monte Carlo experiment is motivated by the study of Rozan (1999). As mentioned above, this study compared the estimated WTP of respondents confronted with one of the two versions of the same questionnaire: one with a low amount for the first bid, and one with a high amount for the first bid. To remain as close as possible to this study, we generate the data set in a similar way. For simplicity, we introduce no explanatory variable, but only a constant in the model. We generate a set of 1000 observations of a normally distributed WTP, $Y_{i 0}^{*}$, with mean 5 and variance 1 , and we set $\sigma_{1}=\sigma_{2}=\sigma=\sqrt{1,36}$ and $\sigma_{12}=0.4,5$ The initial bid, $b_{i}^{1}$, takes values 4 and 6 with equal probabilities, to correspond to the two versions of the questionnaire of Rozan (1999). $Y_{i 1}^{*}$ is constructed as $(1-\lambda) Y_{i 0}^{*}+\lambda b_{i}^{1}+\eta_{i}$ with $\lambda=0.4$ and $\eta_{i} \sim N(0,1)$. The resulting value for $\sigma_{v}$ is equal to $1.36 .{ }^{6}$ For each individual, we generate the response $R_{1}\left(b_{i}^{1}\right)$, which is equal to 1 if $Y_{1 i}^{*}>b_{i}^{1}$ and 0 otherwise. The next set of bids is generated by multiplying or dividing the initial bid by 1.5 depending on the answer to the first question. Thus the data are generated

\footnotetext{
${ }^{3}$ The method used to investigate identification consists in studying the rank of submatrices of first derivatives of the vector of the coefficients of the reduced form of the model with respect to the parameters of interest (see Appendix).

${ }^{4}$ The value for the variance $\sigma$ is obtained as $\sigma^{2}=(1-\lambda)^{2}+1=0.36+1=1.36$.

${ }^{5}$ Note that the restriction $\sigma_{12}=0$ is not imposed in estimation. Imposing it would make the estimation of $\sigma_{v}$ redundant.

${ }^{6}$ The value for the variance $\sigma_{v}$ is obtained as $\sigma_{v}^{2}=0.36 * 1.36+1.36=1.85$.
} 
in the same way as in the survey. Results for 1000 replications are summarized by kernel density estimates for the distribution of each parameter. Summary statistics are given in Table 1.

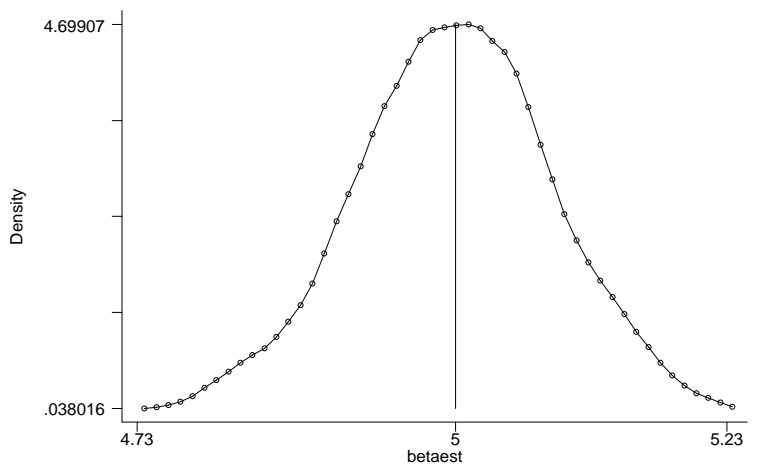

Figure 1: Kernel density estimates of the distribution of the $\beta$ parameter.

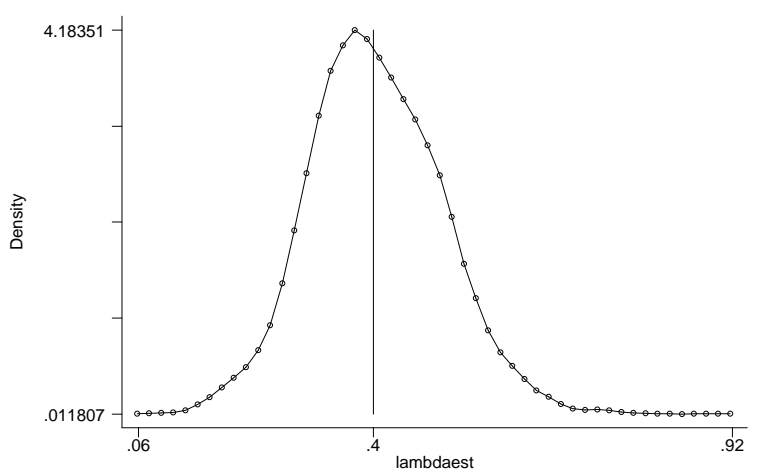

Figure 2: Kernel density estimates of the distribution of the $\lambda$ parameter.

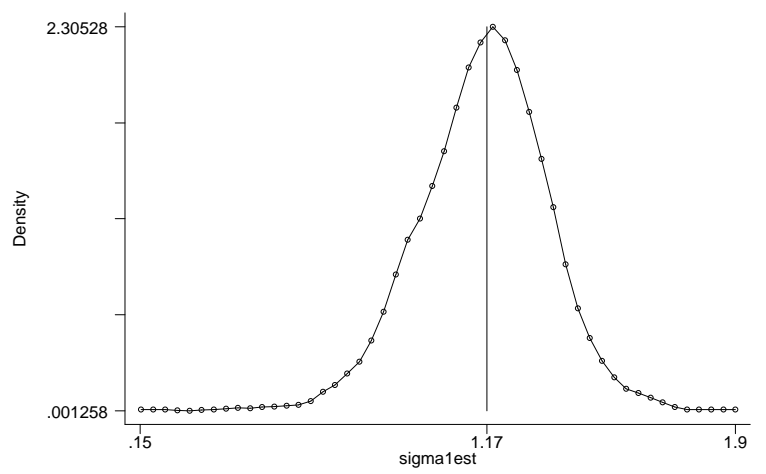

Figure 3: Kernel density estimates of the distribution of the $\sigma$ parameter. 


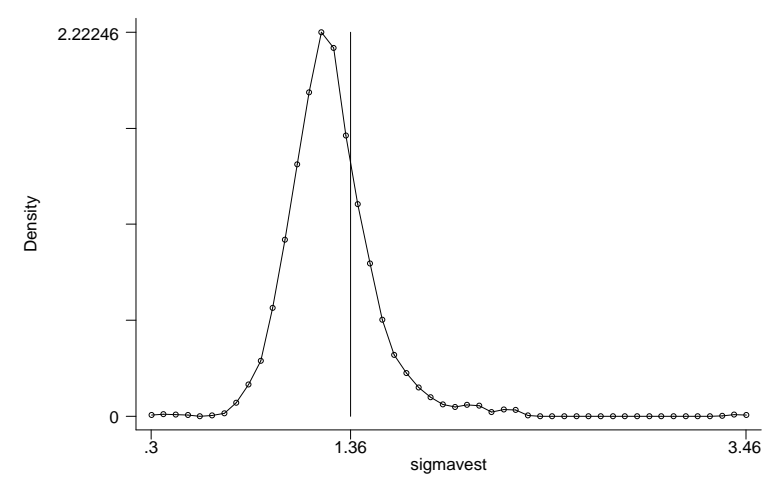

Figure 4: Kernel density estimates of the distribution of the $\sigma_{v}$ parameter.

Table 1: Monte Carlo Simulations for 1000 replications

\begin{tabular}{lcccc}
\hline \hline & $\beta$ & $\lambda$ & $\sigma$ & $\sigma_{v}$ \\
\hline Mean & 4.998 & .405 & 1.159 & 1.256 \\
Standard Error & 0.082 & 0.098 & 0.187 & 0.233 \\
\hline \hline
\end{tabular}

True values are $\beta=5, \lambda=0.4, \sigma=1.17, \sigma_{v}=1.36$

Figures 1 to 4 show the kernel density estimates of the four parameters of the model. The estimates are very closed to the true value of the parameters. This Monte Carlo experiment shows that we can easily estimate the anchoring effect in a model where the starting point bias is supposed to be induced directly by the first response and the successive bids of the model.

\section{Empirical Analysis}

The CV survey used in this paper to analyze the anchoring effect was conducted in Strasbourg (France) in January 1998. It was designed in order to evaluate the minor morbidity costs induced by air pollution. ${ }^{7}$ The costs are defined as the selfmedication charges, as well as the pain and the suffer induced by the light symptoms. An original epidemiological study on light symptoms (Eilstein et al., 1999) implemented in Strasbourg confirmed the existence of a statistically significant shortterm link (0 to 5 days) between air pollution and light symptoms (itchy eyes, runny nose, sore throat, respiratory disorders, headaches). Therefore, the same list of light symptoms has been used for the contingent survey. The whole population

\footnotetext{
${ }^{7}$ See Rozan $(2000,2001)$ for a more detailed overview of this case study.
} 
can be concerned with these symptoms, because they are light and very common. The selected sample is representative of the population residing within the Greater Strasbourg Area (422,849 inhabitants in 1998), per age, gender, socio-professional group, household size and residence quota. The survey took the form of 1000 faceto-face interviews with passers-by. Respondents were told that the symptoms are due to air pollution. In a first step, individuals had to say whether or not they accepted to participate in a program improving air quality. Only those who accepted to participate in the program gave their WTP (after a dichotomous choice with follow-up questioning). The elicitation question format is the closed-ended question with follow-up and at the end an open-ended question allows respondents to reveal their WTP. There were two versions of the closed-ended question with follow-up, the first one began with an amount of $50 \mathrm{FF}$ and the second one with an amount of $2000 \mathrm{FF}$.

Here we restrict attention to people willing to participate in the program, that is to say only 55,3\% of the whole sample. Rozan (1999) found no evidence of a resulting selection bias. Moreover, we analyze the subsample of non-smokers here, as Rozan (1999) found that pooling the non-smoker and smoker subsamples was not tenable. Rozan (2000) also investigated the joint decision to participate or not in the program, and to smoke or not, using a bivariate probit model and a simultaneous probit model (along the lines of Genier and Jacobzone, 1996) and found no significant correlation between the two variables, given the set of exogenous regressors.

A first analysis (see Rozan, 1999), based on the WTP amount given at the end of the closed-ended questions showed that there is indeed an anchoring effect. The mean WTP obtained with the "high" version is significantly higher than the mean WTP obtained with the "low" version. Furthermore, the version indicator is a significant explanatory variable of the WTP amount. We use the same specification for the regressors as Rozan (2000). ${ }^{8}$ There are 313 observations for the non-smoker subsample. We estimate not only the model described in Section 2 but also the onebid and two-bid models, in order to compare these models. These two last models assume anchoring bias away. Following the contingent valuation literature (see, e.g, Cameron and James, 1987; Hanemann et al., 1991), those models are probit-type estimated by direct maximization of likelihoods.

\footnotetext{
${ }^{8}$ Only some of the explanatory variables are presented in Table 2 , those which are significant.
} 
The variables are only dummy variables, which describe the characteristics of the individuals. The variable are described in the Appendix. 
Table 2: Parameter estimates of the model

\begin{tabular}{|c|c|c|c|}
\hline & $1 \mathrm{bid}$ & 2 bids & Anchoring \\
\hline \multirow[t]{2}{*}{ Scenario } & 80.81 & 103.96 & 106.23 \\
\hline & $(121.52)$ & $(95.48)$ & $(122.27)$ \\
\hline \multirow{2}{*}{$\begin{array}{l}\text { Itchy eyes }+ \text { runny nose }+ \\
\text { respiratory disorders. }\end{array}$} & 141.29 & 557.92 & 63.31 \\
\hline & $(485.19)$ & $(253.65)$ & $(545.92)$ \\
\hline \multirow{2}{*}{$\begin{array}{l}\text { Itchy eyes }+ \text { sore throat }+ \\
\text { respiratory disorders. }\end{array}$} & -237.70 & -725.41 & -181.58 \\
\hline & $(478.49)$ & $(256.32)$ & $(539.30)$ \\
\hline \multirow{2}{*}{$\begin{array}{l}\text { Earache }+ \text { coughing }+ \\
\text { headaches }\end{array}$} & 629.49 & 672.07 & 620.50 \\
\hline & $(277.08)$ & $(225.80)$ & $(315.86)$ \\
\hline \multirow[t]{2}{*}{ Informed } & -591.71 & -186.42 & -581.26 \\
\hline & $(160.17)$ & $(143.18)$ & $(205.22)$ \\
\hline \multirow[t]{2}{*}{ ASPA } & 301.21 & 223.53 & 296.70 \\
\hline & $(129.08)$ & $(98.29)$ & $(151.06)$ \\
\hline \multirow[t]{2}{*}{$10000 \mathrm{FF} \leq$ wages $<12500 \mathrm{FF}$} & 314.28 & 222.73 & 269.02 \\
\hline & $(264.53)$ & $(201.94)$ & $(276.93)$ \\
\hline \multirow[t]{2}{*}{$12500 \mathrm{FF} \leq$ wages $<15000 \mathrm{FF}$} & 475.95 & 341.31 & 441.65 \\
\hline & $(225.21)$ & $(182.17)$ & $(259.25)$ \\
\hline \multirow[t]{2}{*}{$15000 \mathrm{FF} \leq$ wages $<20000 \mathrm{FF}$} & 161.76 & 106.69 & 125.37 \\
\hline & $(218.33)$ & $(179.84)$ & $(224.02)$ \\
\hline \multirow[t]{2}{*}{ wages $\geq 20000 \mathrm{FF}$} & 601.84 & 621.42 & 548.07 \\
\hline & $(220.26)$ & $(174.37)$ & (260.61) \\
\hline \multirow[t]{2}{*}{ Constant } & 1766.63 & 1284.07 & 1730.14 \\
\hline & $(244.53)$ & $(211.84)$ & $(493.01)$ \\
\hline \multirow[t]{2}{*}{$\hat{\sigma}$} & 422.32 & 524.73 & 155.58 \\
\hline & $(49.53)$ & $(33.54)$ & (65.97) \\
\hline \multirow[t]{2}{*}{$\hat{\lambda}$} & & & .629 \\
\hline & & & $(.151)$ \\
\hline \multirow[t]{2}{*}{$\hat{\sigma}_{v}$} & & & 253.35 \\
\hline & & & (93.54) \\
\hline
\end{tabular}

Standard errors in parentheses; bold entry indicates significance at the $5 \%$ level.

Scenario $=1$ if the individual was confronted with the first sequence of the questionnaire, 0 otherwise, Informed $=1$ if the individual is informed about the air quality in his town, 0 otherwise and ASPA $=1$ if the individual knows the ASPA (Air-quality monitiring organisation), 0 otherwise. 
Table 2 reports the results for three model specifications: the referendum format (1 bid), the dichotomous choice with follow-up question (2 bids) and the model including a modelisation of the anchoring bias. However, in the CV study (Rozan, 1999), the elicitation format was not conventional. Indeed, the closed-ended question with follow-up was used, but the choice of the bids was designed to bracket the WTP, in an attempt to avoid the anchoring effect. As already mentioned, the first bid was either very low, in the first version (50 FF), or very high (2000 FF), in the second one. Depending on the first answer, the second bid was very high (2000 FF) for the first version (respectively very low (50 FF) for the second version), if the individual answered "yes" (respectively "no") to the first question. At the most, people had to answer six closed-ended questions. Due to this choice of bids, the efficiency gain is not very substantial, as Hanemann et al.(1991) have found. This explains why we do not observe a great reduction of the standard errors between the one-bid and two-bids models.

Focusing on the anchoring model (column 3 in Table 2), we observe that the anchoring coefficient $(\lambda)$ is statistically significant at a $5 \%$ level, with $\lambda=0.629$. Thus the anchoring effect appears to play a significant role in this study, and we could suppose that the first proposed bid has a direct influence on the individual's WTP. People are influenced by the first proposed bid, before answering the first question. This level of anchoring effect is very high if we compare it to the study of Herriges and Shogren (1996). Indeed, these authors observed no evidence of the anchoring effect on the population of local residents but a significant anchoring effect $(\lambda=0.36)$ on the recreationists. This could be explained by the specification of our model, which is different than the model used by Herriges and Shogren (1996). We do not introduce the anchoring effect in the model after the response to the first question, but directly at the beginning of the questionnaire.

For the results of the anchoring effect model, we notice that our estimates are closer to the results of the one-bid model than to the two-bids model. This is the case for all coefficients except for the estimated dispersion coefficient $(\hat{\sigma})$. This coefficient is much smaller than in the first bid model. When we control for anchoring, id est when we specify a model with an anchoring coefficient, we observe also, that the estimated standard errors of all estimated coefficients are larger than those in the first bid model. 


\section{Conclusion}

During the last decade many studies (Kanninen, 1995, Greene et al., 1998) found strong anchoring effects induced by dichotomous choice with follow-up. As far as we know, only Herriges and Shogren (1996) proposed a model of the starting point bias. But their model only takes into account the effect of the first bid on the second response. This paper provides a new approach to estimating the starting point bias in the follow-up format. We propose a model based on the hypothesis that the anchoring effect is not only due to the first bid, but is induced by all bids of the questionnaire.

We restrict attention to a double bounded model and estimate the anchoring effect in a Monte Carlo experiment, and also use real data based on a CV survey on health effects due to air pollution. Our empirical analysis shows that the questionnaire conducted by Rozan (1999) leads to a starting point bias as found in earlier CV studies.

There are two mains directions for future research. First, this model is restricted to a double-bounded questionnaire and does not take into account a higher number of bids. Thus it is of interest to extent this method to a triple or multiple bounded dichotomous choice model. In particular this should allow us to test whether or not the anchoring effect is the same for all additional bids. Second, our model supposes that the anchoring process is the same for all individuals. It would be interesting to test this assumption against the alternative where anchoring effects across individuals are different.

\section{References}

Arrow, K., R. Solow, P. Portney, E. Leamer, R. Radner, and H. SchuMAN (1993): Report of the NOAA Panel on Contingent Valuation. Resources for the Future, Washington DC.

Cameron, T. A., And M. James (1987): "Efficient Estimation Methods for "Closed-Ended Contingent Valuation Surveys," The review of Economics and Statistics, pp. 269-276. 
Cameron, T. A., And J. Quiggin (1994): "Estimation using Contingent Valuation Data from a "Dichotomous Choice with Follow-Up" Questionnaire," Journal of Environmental Economics Management, 24, 218-234.

EILstein, D., AND AL (1999): "Etude RAMSES I : Investigaion sur Les Relations Entre la Pollution Atmosphérique et la Survenue de Symptômes Cliniques Recueillis Par Le Réseau Alsace Des Médecins Pour la Surveillance Des Relations Entre L'environnement et la Santé En 1996 et 1997," Discussion paper, GRES Alsace.

Genier, P., and S. Jacobzone (1996): "Comportement de Prévention, Consommation D'alcool et Tabagie: Peut on Parler D'une Gestion Globale Du Capital Santé ? Une Modélisation Microéconométrique Empirique.," Discussion paper, Document de travail INSEE.

Greene, D., K. E. Jacowitz, D. Kahneman, and D. McFadden (1998): "Referendum Contingent Valuation, Anchoring, and Willingness-to-Pay for Public Goods," Resource and Energy Economics, 20, 85-116.

Hanemann, M., J. Loomis, and B. Kanninen (1991): "Statistical Efficiency of Double-Bounded Dichotomous Choice Contingent Valuation," American Journal of Agricultural Economics, pp. 1255-1263.

Hausman, J. (1993): Contingent Valuation: A Critical Assessment. North-Holland.

Herriges, J. A., And J. F. Shogren (1996): "Starting Point Bias in Dichotomous Choice Valuation with Follow-Up Questioning," Journal of Environmental Economics Management, 30, 112-131.

Kanninen, B. J. (1995): "Bias in Discrete Response Contingent Valuation," Journal of Environmental Economics Management, 28, 114-125.

LAngford, H. I., I. J. Bateman, And H. LAngford (1996): "A Multilevel Modelling Approach to Triple-Bounded Dichotomous Choice Contingent Valuation," Environmental and Resource Economics, 7, 197-211.

Mitchell, R. C., And R. T. Carson (1989): Using Surveys to Value Public Goods: The Contingent Valuation Method. Johns Hopkins University Press, Baltimore, MD.

O'Connor, R. M., M. Johanesson, and P. O. Johansson (1999): "StatedPreferences, Real Behaviour and Anchoring: Some Empirical Evidence," Environmental and Resource Economics, 13, 235-248.

Rozan, A. (1999): Evaluation Des Bénéfices de Santé D'une Amélioration de la Qualitéde L'air : L'exemple de la Région Strasbourgeoise. Ph.D thesis. Thèse pour le doctorat de Sciences Economiques, Université Louis Pasteur, Strabourg I. 
(2000): "Une Évaluation Économique Des Bénéfices de Morbidité Induits Par Une Amélioration de la Qualité de l'Air," Economie et Prévision, (143-144), 247-259.

(2001): "How to Measure Health Costs Induced by Air Pollution," Schweizerische Zeitschrift für Volkswirtchaft und Statistik, (Swiss Journal of Economics and Statistics), 137(1), 103-116. 


\section{Appendix}

\section{Reduced Form of the model}

The reduced form associated with the model (2.2) and (2.5) is defined as

$$
\begin{gathered}
Z_{i 1}^{*}=\frac{Y_{i 1}^{*}-b_{i}^{1}}{\sigma_{1}}=\underbrace{\frac{(1-\lambda) \beta}{\sigma_{1}}}_{\pi_{12}}-\underbrace{\frac{(1-\lambda)}{\sigma_{1}}}_{\pi_{11}} b_{i}^{1}+\frac{u_{i 1}}{\sigma_{1}} \\
Z_{i 2}^{*}=\frac{Y_{i 2}^{*}-b_{i}^{2}}{\sigma_{v}}=\underbrace{\frac{(1-\lambda)^{2} \beta}{\sigma_{v}}}_{\pi_{23}}+\underbrace{\frac{\lambda(1-\lambda)}{\sigma_{v}}}_{\pi_{21}} b_{i}^{1}-\underbrace{\frac{(1-\lambda)}{\sigma_{v}}}_{\pi_{22}} b_{i}^{2}+\frac{v}{\sigma_{v}}
\end{gathered}
$$

The observed binary variable is defined as:

$$
Z_{i k}=\mathbb{1}\left[Z_{i k}^{*}>0\right] \quad k=1,2 .
$$

Thus, when we estimate the model with maximum likelihood we estimate the five coefficients $\pi_{11}, \pi_{12}, \pi_{21}, \pi_{22}, \pi_{23}$.

\section{Log-likelihood of the model}

Let $h_{i 1}=\frac{b_{i}^{1}-(1-\lambda) X_{i} \beta-\lambda b_{i}^{1}}{\sigma_{1}}$ and $h_{i 2}=\frac{b_{i}^{2}-(1-\lambda)^{2} X_{i} \beta-\lambda(1-\lambda) b_{i}^{1}-\lambda b_{i}^{2}}{\sigma_{v}}$. The bivariate density function is then defined as :

$$
g\left(h_{i 1}, h_{i 2}\right)=\frac{1}{2 \pi \sigma_{1} \sigma_{v} \sqrt{\left(1-\rho^{2}\right)}} \exp \left(-\frac{1}{2\left(1-\rho^{2}\right)}\left[h_{i 1}^{2}-2 \rho h_{i 1} h_{i 2}+h_{i 2}^{2}\right]\right)
$$

The log-likelihood of the model defined by the equations (2.3) and (2.5) is written as :

$$
\begin{aligned}
\log L & =\sum_{i}\left(\left(R_{1}\left(b_{i}^{1}\right) R_{2}\left(b_{i}^{2}\right)\right) \log \left[\int_{h_{i 1}}^{\infty} \int_{h_{i 2}}^{\infty} g\left(h_{i 1}, h_{i 2}\right) d h_{i 1} d h_{i 2}\right]\right. \\
& +\left(1-R_{1}\left(b_{i}^{1}\right)\right)\left(R_{2}\left(b_{i}^{2}\right)\right) \log \left[\int_{-\infty}^{h_{i 1}} \int_{h_{i 2}}^{\infty} g\left(h_{i 1}, h_{i 2}\right) d h_{i 1} d h_{i 2}\right] \\
& +\left(1-R_{1}\left(b_{i}^{1}\right)\right)\left(1-R_{2}\left(b_{i}^{2}\right)\right) \log \left[\int_{-\infty}^{h_{i 1}} \int_{-\infty}^{h_{i 2}} g\left(h_{i 1}, h_{i 2}\right) d h_{i 1} d h_{i 2}\right] \\
& \left.+\left(R_{1}\left(b_{i}^{1}\right)\right)\left(1-R_{2}\left(b_{i}^{2}\right)\right) \log \left[\int_{h_{i 1}}^{\infty} \int_{-\infty}^{h_{i 2}} g\left(h_{i 1}, h_{i 2}\right) d h_{i 1} d h_{i 2}\right]\right) .
\end{aligned}
$$




\section{Description of the variables}

Itchy eyes, runny nose respiratory disorders,

Itchy eyes, sore throat, respiratory disorders

Earaches, Cough

Headache
$=1$ if the individual or a member of his family has suffered from itchy eyes, runny nose and respiratory disorders, 0 otherwise.

$=1$ if the individual or a member of his family has suffered from itchy eyes, sore throat and respiratory disorders, 0 otherwise

$=1$ if the individual or a member of his family has suffered from earaches, cough and headache, 0 otherwise

$10000 \mathrm{FF} \leq$ wages $<12500 \mathrm{FF}=1$ if the earnings of the family is between $10000 \mathrm{FF}$ and $12500 \mathrm{FF}, 0$ otherwise.

$12500 \mathrm{FF} \leq$ wages $<15000 \mathrm{FF}=1$ if the earnings of the family is between $12500 \mathrm{FF}$ and $15000 \mathrm{FF}, 0$ otherwise.

$15000 \mathrm{FF} \leq$ wages $<20000 \mathrm{FF}=1$ if the earnings of the family is between $15000 \mathrm{FF}$ and 20000FF, 0 otherwise.

wages $\geq 20000 \mathrm{FF}$

$=1$ if the earnings of the family is bigger then $20000 \mathrm{FF}$, 0 otherwise 
Table 3: Descriptive statistics

\begin{tabular}{ll}
\hline \hline & Mean \\
\hline \hline Scenario & .549 \\
Itchy eyes & .383 \\
Runny nose & .664 \\
Sore throat & .578 \\
Earache & .217 \\
Coughing & .559 \\
Enrouement & .390 \\
Respiratory disorders & .332 \\
Sinusitis & .204 \\
Bronchitis & .195 \\
Headache & .597 \\
Itchy eyes + runny nose + respiratory disorders. \\
Itchy eyes + sore throat + respiratory disorders. \\
Earache + coughing + headaches & .128 \\
Informed & .885 \\
ASPA & .380 \\
wages $<5000 \mathrm{FF}$ & .121 \\
$5000 \mathrm{FF} \leq$ wages $<7500 \mathrm{FF}{ }^{(*)}$ & .147 \\
$7500 \mathrm{FF} \leq$ wages $<10000 \mathrm{FF}$ & .160 \\
$10000 \mathrm{FF} \leq$ wages $<12500 \mathrm{FF}$ & .109 \\
$12500 \mathrm{FF} \leq$ wages $<15000 \mathrm{FF}$ & .134 \\
$15000 \mathrm{FF} \leq$ wages $<20000 \mathrm{FF}$ & .182 \\
wages $\geq 20000 \mathrm{FF}$ & .147 \\
\hline \hline
\end{tabular}

$\left.{ }^{*}\right)$ this income category is considered as the benchmark category. 